\title{
Helium partitioning between the Earth's mantle and the core during the core formation
}

\author{
OZGE OZGUREL ${ }^{1}$ AND RAZVAN CARACAS ${ }^{1,2}$ \\ ${ }^{1}$ University of Oslo \\ ${ }^{2} \mathrm{CNRS}$ \\ Presenting Author: ozge.ozgurel@geo.uio.no
}

Noble gases are geochemical tracers, providing information about the formation of our planet and serving as a record of conditions in Earth history. Each noble gas has at least one stable non-radiogenic isotope, which is residue either of Big Bang or supernovas, and at least one stable radiogenic isotope, product of nuclear decay reactions from unstable heavier isotope of another element. The ratio of non-radiogenic and radiogenic isotopes of the noble gases arriving at the surface are essential to understand processes occurring on various timescale in the Earth history and in the Earth interior.

The isotopic signature of the noble gases in the mid-ocean ridge basalts (MORBs) are different than in the ocean island basalts (OIB) such as in Iceland, Hawaii, Galapagos, Réunion, or Samoa. One such example is the high ${ }^{3} \mathrm{He} /{ }^{4} \mathrm{He}$ ratios observed in OIB, which are explained as a signature of the core, which in this case becomes a hidden geochemical reservoir. Here, we study the Helium partitioning between molten pyrolite and liquid iron, representing proxies for the crystallizing magma ocean and the growing core, respectively.

We employ molecular dynamics simulations based on the density functional theory as implemented in the VASP package. We perform the simulations at several temperatures and pressures that sample the magma ocean adiabat. We explore various theoretical approaches, including putting the two melts in contact, using thermodynamical integration, and free energy calculations. We determine the chemical potential and the chemical affinity of helium, we estimate the amount of core mantle mixing, and we characterize the effect of mixing on the helium partitioning.

We acknowledge support from the Research Council of Norway, project number 223272. RC acknowledges support from the European Research Council under EU Horizon 2020 research and innovation program (grant agreement 681818 - IMPACT) and access to supercomputing facilities via the eDARI gen6368 grants, the PRACE RA4947 grant, and the Uninet2 NN9697K grant. 